\title{
Electronically Tunable Oscillator Utilizing Reinforced Controllable Parameters
}

\author{
LANGHAMMER, L.; ŠOTNER, R.; DOMANSKÝ, O.
}

Proceedings of the 2019 11th International Congress on Ultra Modern Telecommunications and Control Systems and Workshops (ICUMT), pp. 1-5

elSBN: 978-1-7281-5763-4

ISSN: 2157-023X

DOI: https://doi.org/10.1109/ICUMT48472.2019.8970997

Accepted manuscript

(C2019 IEEE. Personal use of this material is permitted. Permission from IEEE must be obtained for all other uses, in any current or future media, including reprinting/republishing this material for advertising or promotional purposes, creating new collective works, for resale or redistribution to servers or lists, or reuse of any copyrighted component of this work in other works. LANGHAMMER, L.; ŠOTNER, R.; DOMANSKÝ, O. "Electronically Tunable Oscillator Utilizing Reinforced Controllable Parameters", 2019 11th International Congress on Ultra Modern Telecommunications and Control Systems and Workshops (ICUMT), 2019.

DOI: 10.1109/ICUMT48472.2019.8970997. Final version is available at 


\title{
Electronically Tunable Oscillator Utilizing Reinforced Controllable Parameters
}

\author{
Lukas Langhammer, Roman Sotner, Ondrej Domansky \\ Faculty of Electrical Engineering and Communication \\ Brno University of Technology \\ Technicka 12, 61600 Brno, Czech Republic \\ xlangh01@stud.feec.vutbr.cz
}

\begin{abstract}
This paper presents a novel solution of an oscillator with electronically adjustable oscillation condition (CO) and frequency of oscillations (FO). Oscillation condition is controlled by current gain and frequency of oscillations is adjustable by transconductance and intrinsic resistance of used active elements. Both $\mathrm{CO}$ and $\mathrm{FO}$ are mutually independent. Moreover, special feature of $\mathrm{CO}$ allows boosting parameter driving FO (transconductance) and then shifting the whole FO range to higher bands. It allows to keep values of passive elements (capacitors especially) in satisfactory range even for higher value of FO. Simulations in PSpice confirms this hypothesis.
\end{abstract}

Keywords-adjustable current gain, electronic control, intrinsic resistance, oscillator, transconductance

\section{INTRODUCTION}

The ability of controllable condition of oscillation (CO) and frequency of oscillation (FO) are very important features of many current oscillator designs and topologies [1]. The practically useful adjustment of CO and FO supposes existence of two independent parameters of the characteristic equation [1]. It means that $\mathrm{CO}$ and $\mathrm{FO}$ are settable without disturbance of each other. The first group of circuit solutions targets on values of passive elements (see [1]-[3] and references cited therein). This type of oscillators is frequently called as single resistance-controlled oscillator (SRCO) [4]. The second group covers oscillators offering ability of the electronic control of their $\mathrm{CO}$ and FO thanks to the usage of electronically controllable active elements (see for example [1], [5]-[11] and references cited therein). The widespread method consists in transconductance $\left(g_{\mathrm{m}}-\right.$ conversion constant between voltage and current) control of FO and $\mathrm{CO}$ [1], [5], [6]. Other possible way of driving utilizes an intrinsic resistance of current input terminals $R_{\mathrm{X}}$ for the adjustability [1]. Methods presented in [7]-[9] combine both above-mentioned parameters in order to achieve electronic controllability of $\mathrm{FO}$ and $\mathrm{CO}$. An example of an oscillator using current gain $B$ to control FO can be found in [10]. A tunability implementing voltage gain $A$ is presented in [11].

\section{STATE-OF-THE-ART}

The tunability of a controllable oscillator supposes existence of active elements with electronically adjustable parameters [1]. High number of solutions employs $g_{\mathrm{m}}$ of the operational transconductance amplifier (OTA) [12], [13] for these purposes. OTAs are parts of many advanced active elements [1], [7]-[9], [12].

Unfortunately, available ranges of $g_{\mathrm{m}}$ value are not favorable in modern CMOS processes (from units

Research described in the paper was supported by Czech Science Foundation project under No. 19-22248S. to hundreds of $\mu \mathrm{S}$ ) [13]. Then, the design for higher operational frequencies (above $1 \mathrm{MHz}$ ) considers also low values of passive elements (resistors and capacitors) as well as high values of $g_{\mathrm{m}}$. It brings significant problems with parasitic properties of the real circuit because values of elements are near to parasitic capacitances (units of $\mathrm{pF}$ ) in high-impedance nodes [14]. Therefore, their impact on the expected value and accuracy of frequency of oscillations has really significant impact (deviations in tens of percent) and cannot be neglected.

A method how to surpass unsuitable ranges of $g_{\mathrm{m}}$-s in the OTA is presented in this paper. It utilizes a topological feature of newly proposed circuit where a term of the numerator of the equation for oscillation frequency is reinforced (boosted) by an additional multiplicative factor. The equation for FO includes $g_{\mathrm{m}}$ parameters in standard case [5], [6]. However, our improvement consists in presence of an additional parameter. This parameter represents integer value of the current gain that can be easily increased by additional output mirrors of CMOS structure. Note that condition of oscillation is not disrupted. Despite its presence also in $\mathrm{CO}$, the value is fixed in the operation because $\mathrm{CO}$ can be driven by the different current gain, not influencing FO. Thus, the parameter works only as multiplicative constant established at the start of the design process. It yields shift of the FO range to higher frequencies whereas range of $g_{\mathrm{m}}$ remains unchanged. We tested this effect by the simulation of standard macromodels of commercially available active elements. It is sufficient in order to confirm possible design methodology that can be generalized for any specific type of OTA and other active elements.

TABLE I. COMPARISON OF LINEARLY TUNABLE OSCILLATORS (PHASE SHIFT $\pi / 4$ )

\begin{tabular}{|c|c|c|c|c|c|}
\hline 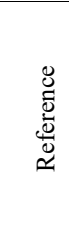 & 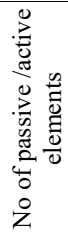 & 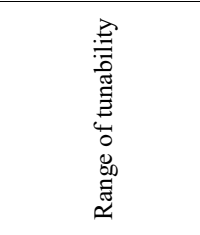 & 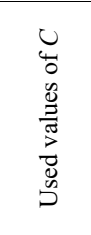 & 㓠 & 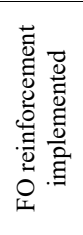 \\
\hline [15] & $4 / 1$ & $1.3 \rightarrow 7.39 \mathrm{MHz}$ & $30 \mathrm{pF}$ & $5.7: 1$ & No \\
\hline [16] & $2 / 2$ & $0.4 \rightarrow 1.8 \mathrm{MHz}$ & $100 \mathrm{pF}$ & $4.5: 1$ & No \\
\hline [17] & $2 / 3$ & $1.1 \rightarrow 3.3 \mathrm{MHz}$ & $68 \mathrm{pF}$ & $3: 1$ & No \\
\hline [18] & $2 / 1$ & $0.15 \rightarrow 1.9 \mathrm{MHz}$ & $100 \mathrm{pF}$ & $13: 1$ & No \\
\hline $\begin{array}{l}\text { This } \\
\text { work }\end{array}$ & $3 / 4$ & $0.21 \rightarrow 2.02^{\mathrm{a}} \mathrm{MHz}$ & $72 \mathrm{pF}$ & $9.6: 1$ & No \\
\hline $\begin{array}{l}\text { This } \\
\text { work }\end{array}$ & $3 / 4$ & $0.21 \rightarrow 2.07^{\mathrm{b}} \mathrm{MHz}$ & $102 \mathrm{pF}$ & $9.9: 1$ & Yes \\
\hline $\begin{array}{l}\text { This } \\
\text { work }\end{array}$ & $3 / 4$ & $0.21 \rightarrow 2.09^{\mathrm{c}} \mathrm{MHz}$ & $125 \mathrm{pF}$ & $10: 1$ & Yes \\
\hline
\end{tabular}


The proposed circuitry generates two waveforms having $\pi / 4$ phase shift. The selected solutions concerning similar types of the oscillator [15]-[18] are compared in Table I. None of the previously presented topologies provides the feature of the reinforcement of FO.

\section{OSCILLATOR PROPOSAL}

The proposed structure (Fig. 1) comprises one voltage differencing current conveyor (VDCC) [15], two currentmode multipliers implemented by EL2082 devices [19], single resistor, two voltage buffers and two capacitors. The internal structure of the VDCC element is depicted in Fig. 2. It was implemented by three types of commercially available active elements: LT1228 device [20] realizing the function of an operational transconductance amplifier (OTA) [12], [13]. It follows the relationship $i_{\mathrm{OUT}}=g_{\mathrm{m}}\left(v_{\mathrm{IN}+}-v_{\mathrm{IN}}\right)$. The second type of element is a current feedback operational amplifier (CFOA) [1], [12] implemented by AD844 device [21]. This active element can be described by the relation $i_{\text {OUT }}=i_{\mathrm{IN}}$. The remaining active element in the VDCC structure is a current-mode multiplier (CM) realized by EL4083 [22] device. The behavior of the current multiplier (EL4083 and EL2082 devices) is characterized by $i_{\text {OUT } \pm}= \pm B i_{\mathrm{IN}}$.

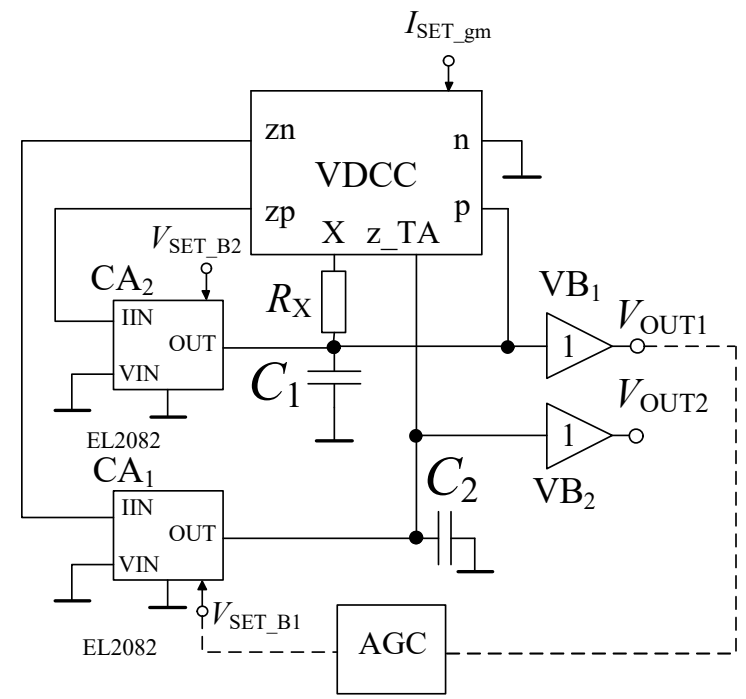

Fig. 1. Circuit diagram of the proposed oscillator.

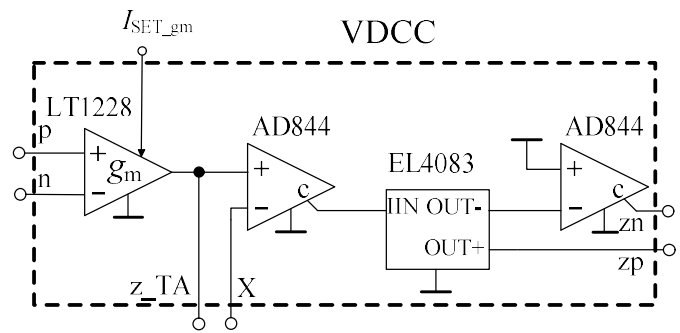

Fig. 2. CG-VDCC element realization using commercially available devices

The characteristic equation of the oscillator takes form:

$$
s^{2}+s \frac{C_{2}+B_{1} C_{1}-B_{2} C_{2}}{R_{X} C_{1} C_{2}}+\frac{g_{m}\left(B_{2}-1\right)}{R_{X} C_{1} C_{2}}=0 .
$$

The relations for the condition of oscillations and frequency of oscillations are given as:

$$
\begin{gathered}
B_{2} \geq B_{1} \frac{C_{1}}{C_{2}}+1, B_{1} \leq B_{2} \frac{C_{2}}{C_{1}}-\frac{C_{2}}{C_{1}}, \\
f_{0}=\frac{1}{2 \pi} \sqrt{\frac{g_{m}\left(B_{2}-1\right)}{C_{1} C_{2} R_{X}}} .
\end{gathered}
$$

Based on discussion of (2), (3) and (4), CO can be controlled electronically by current gain $B_{1}\left(B_{2}=\right.$ constant $)$, when FO can be also electronically tuned solely by the transconductance $g_{\mathrm{m}}$ (nonlinear control of $f_{0}$ - controlling parameter is under the root), solely by the intrinsic resistance $R_{\mathrm{X}}$ (nonlinear control of $f_{0}$ ), or by both parameters simultaneously when the following condition $g_{\mathrm{m}}=1 / R_{\mathrm{X}}$ (linear control of $f_{0}-$ can be extracted from the root since $g_{\mathrm{m}}$ $\left.=1 / R_{\mathrm{X}}\right)$ is fulfilled. Note that $B_{2}$ can be used for boosting of $g_{\mathrm{m}}$ value that causes an increase of FO without modification of the OTA maximal available $g_{\mathrm{m}}$ value. It is useful because $g_{\mathrm{m}}$ values in modern CMOS technologies are quite limited in general (hundreds of $\mu \mathrm{S}$ ).

The CO can be simplified if the values of capacitors $C_{1}$ and $C_{2}$ are supposed being equal. $C O$ turns into: $B_{2} \geq B_{1}+1$, $B_{1} \leq B_{2}-1$. The initial design supposes that $B_{2}$ is set to the intended value $\left(B_{2}=2\right)$ and then it must remain fixed during the further operation (in order to avoid the disturbance of FO). is:

The ratio of amplitudes between outputs $V_{\text {OUT1 }}$ and $V_{\text {OUT2 }}$

$$
\frac{V_{\text {OUT } 1}}{V_{\text {OUT } 2}}=\frac{1-B_{2}}{1-B_{2}+s C_{1} R_{X}} .
$$

If we suppose equality of both capacitors $C_{1}=C_{2}=C$, then the ratio of amplitudes between outputs is obtained as:

$$
\frac{V_{\text {OUT } 1}}{V_{\text {OUT } 2}}=\frac{1+j \sqrt{g_{m} R_{X}}}{\sqrt{g_{m} R_{X}}+1} .
$$

The phase shift between outputs $\left(\sqrt{ }\left(g_{\mathrm{m}} \cdot R_{\mathrm{X}}\right)=1\right)$ achieves:

$$
\frac{V_{\text {OUT } 1}}{V_{\text {OUT } 2}}=\frac{\sqrt{2}}{2} e^{\frac{\pi}{4} j} .
$$

Thus, the theoretical ratio of the output amplitudes is $V_{\text {OUT2 }}=1.4 V_{\text {OUT1 }}$ and the phase shift between outputs is $45^{\circ}$.

\section{CIRCUIT VERIFICATION}

The proposed oscillator was verified using PSpice simulations. The simulations were carried out using available behavioral models of used active elements. Selected simulations were included in the paper for illustrations. Values of capacitors were set to $C_{1}=C_{2}=C=220 \mathrm{pF}$. The tested range of values of $g_{\mathrm{m}}$ was from $0.1 \mathrm{mS}$ to $1 \mathrm{mS}$. Similarly, intrinsic resistance $R_{\mathrm{X}}$ has been set in range from $10 \mathrm{k} \Omega$ to $1 \mathrm{k} \Omega$. In case of the linear control of FO both these parameters were adjusted simultaneously when $R_{\mathrm{X}}=1 / g_{\mathrm{m}}$. Current gains were set as follows: $B_{2}=2$ (remains unchanged), $B_{1} \leq 1$ (controlled electronically by automatic gain control (AGC) circuit in order to fulfill $\mathrm{CO}$ ). 
Fig. 3 shows the output responses (time domain) of outputs $V_{\text {OUT1 }}$ and $V_{\text {OUT2 }}$ for $g_{\mathrm{m}}=1 \mathrm{mS}$ and $R_{\mathrm{X}}=1 \mathrm{k} \Omega$. Then, the theoretical $f_{0}$ reaches $723 \mathrm{kHz}$. The simulation results yield $684 \mathrm{kHz}$. The theoretical range of $f_{0}$ depending on $g_{\mathrm{m}}$ values $\left(g_{\mathrm{m}}=0.1 \rightarrow 1 \mathrm{mS}, B_{2}=2, B_{1} \leq 1\right)$ was from $229 \mathrm{kHz}$ to $723 \mathrm{kHz}$ (nonlinear control by $g_{\mathrm{m}}$ only). Values obtained from simulations yield range from $219 \mathrm{kHz}$ to $684 \mathrm{kHz}$. The same behavior can be obtained for $f_{0}$ tuned by $R_{\mathrm{X}}$ value $\left(R_{\mathrm{X}}=10 \rightarrow 1 \mathrm{k} \Omega\right)$.

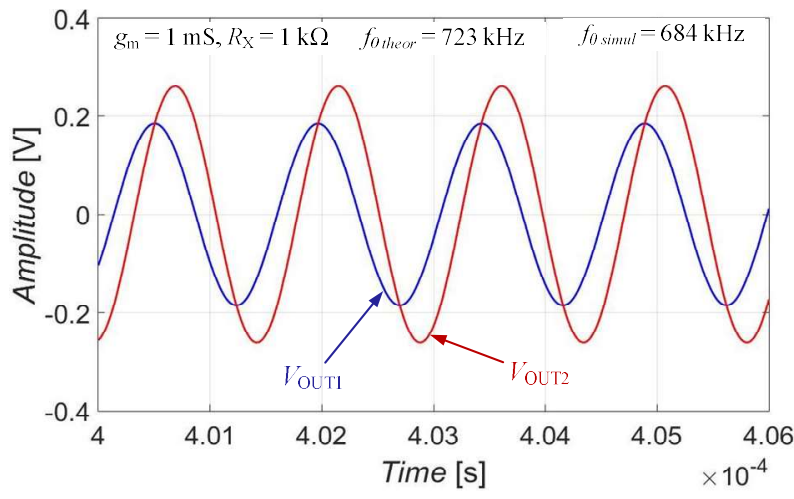

Fig. 3. Output responses of the oscillator (time domain) for $B_{2}=2, B_{1} \leq 1$.

Fig. 4 compares the theoretical and simulated dependence of the $f_{0}$ on $g_{\mathrm{m}}$ or $R_{\mathrm{X}}$. Both dependencies are almost identical. The differences between the theory and obtained results are getting greater as the $f_{0}$ increase especially due to the impact of parasitic capacitances in high-impedance nodes of $C_{1}$ and $C_{2}$.

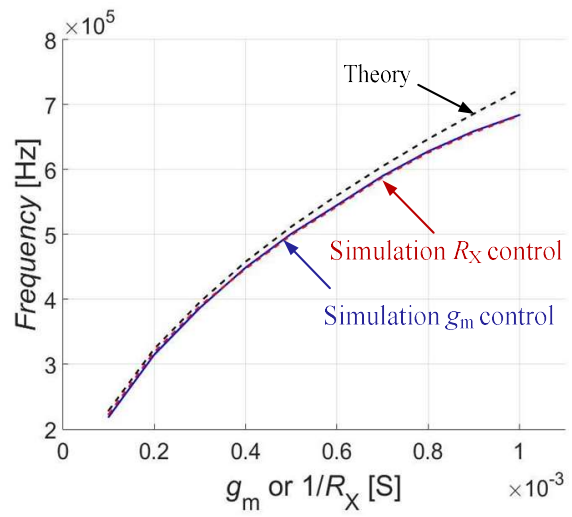

Fig. 4. Dependency of the nonlinear FO control on $g_{\mathrm{m}}$ or $R_{\mathrm{X}}\left(B_{2}=2\right)$.

Fig. 5 shows the linear dependence of the FO on driving parameters (simultaneously varied $g_{\mathrm{m}}$ and $R_{\mathrm{X}}$ when their ratio is $\left.g_{\mathrm{m}}=1 / R_{\mathrm{X}}\right)$ for same case as before $\left(B_{2}=2, B_{1} \leq 1\right)$. The theoretical range of obtainable $f_{0}$ in this case is from $72 \mathrm{kHz}$ to $723 \mathrm{kHz}$. The simulated results provide range from $73 \mathrm{kHz}$ to $684 \mathrm{kHz}$. It can be seen that the linear control of FO (when controlling $g_{\mathrm{m}}$ and $R_{\mathrm{X}}$ simultaneously) provide wider range of obtainable FO: $219 \mathrm{kHz}$ to $684 \mathrm{kHz}$ $\left(f_{0 \text { max }} / f_{0 \text { min }}\right.$ range of 3.2) in case of non-linear control in comparison to $73 \mathrm{kHz}$ to $684 \mathrm{kHz}\left(f_{0 \_ \text {max }} / f_{0 \_ \text {min }}\right.$ range of 9.4) for the linear control.

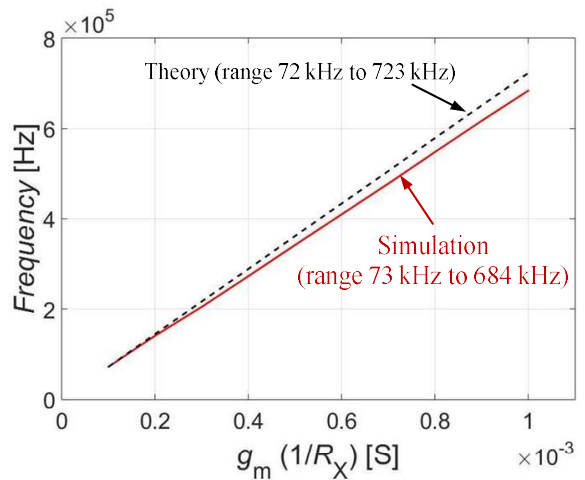

Fig. 5. Dependency of the linear FO control on used values of $g_{\mathrm{m}}$ and $R_{\mathrm{X}}$ $\left(g_{\mathrm{m}}=1 / R_{\mathrm{X}}\right)$

As mentioned earlier, the proposed oscillator offers a special feature of FO reinforcement (shifting the FO range to higher frequencies) thanks to its specific characteristic equation when $\mathrm{CO}$ and the mutual independence of $\mathrm{CO}$ and FO remains fulfilled. The current gain $B_{2}$ set to 3 (and $\left.B_{1} \leq 2\right)$ modifies the relation for $\mathrm{FO}$ (4) to form $f_{0}=1 / 2 \pi \cdot\left(2 g_{\mathrm{m}} /\left(C_{1} C_{2} R_{\mathrm{X}}\right)\right)^{1 / 2}$ whereas CO still being valid. The theoretical range of $f_{0}$ then shifts to $f_{0}=324 \mathrm{kHz}$ $\rightarrow 1023 \mathrm{kHz}$ (nonlinear control) for $g_{\mathrm{m}}=0.1 \rightarrow 1 \mathrm{mS}$. The simulated values fall into range $322 \mathrm{kHz}$ and $923 \mathrm{kHz}$. Fig. 6 compares simulated results of standard ( $B_{2}=2$, Fig. 4$)$ and boosted $\left(B_{2}=3\right)$ FO tuning range.

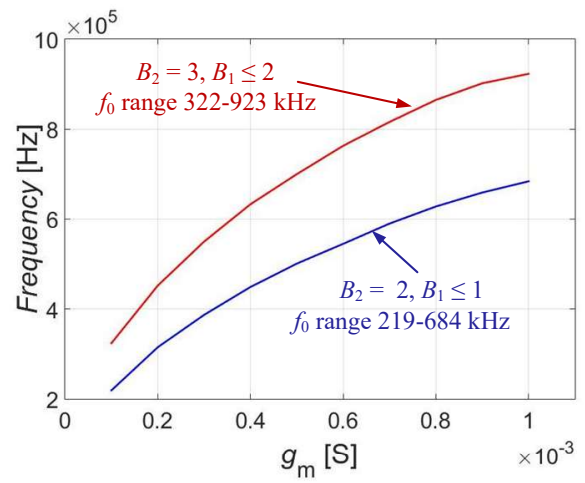

Fig. 6. Dependency of the nonlinear FO control on $g_{\mathrm{m}}$ for $B_{2}=2$ and $B_{2}=3$.

Fig. 7 illustrates the output responses (time domain) of $V_{\text {OUT1 }}$ and $V_{\text {OUT2 }}$ for values of $g_{\mathrm{m}}$ and $R_{\mathrm{X}}$ set as in Fig. 3 $\left(g_{\mathrm{m}}=1 \mathrm{mS}\right.$ and $\left.R_{\mathrm{X}}=1 \mathrm{k} \Omega\right)$ and $B_{2}=3 \quad\left(B_{1} \leq 2\right)$. The theoretical $f_{0}$ is equal to $1023 \mathrm{kHz}$. The obtained $f_{0}$ for outputs was $923 \mathrm{kHz}$.

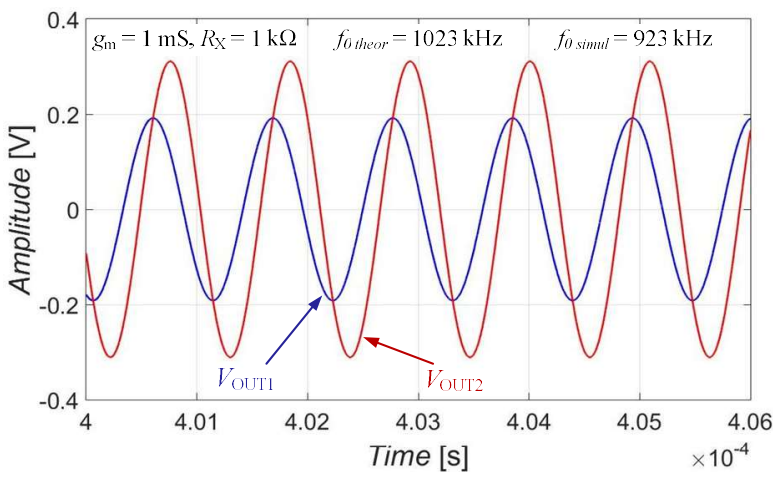

Fig. 7. Output responses of the oscillator (time domain) for $B_{2}=3, B_{1} \leq 2$. 
Comparison of the dependence of FO on the values of $g_{\mathrm{m}}$ and $R_{\mathrm{X}}\left(R_{\mathrm{X}}=1 / g_{\mathrm{m}}\right)$ for the setting $B_{2}=2, B_{1} \leq 1$, $C_{1,2}=72 \mathrm{pF}, B_{2}=3, B_{1} \leq 2, C_{1,2}=102 \mathrm{pF}$ and $B_{2}=4, B_{1} \leq 3$, $C_{1,2}=125 \mathrm{pF}$ (when $g_{\mathrm{m}}$ is changed in range from $0.1 \mathrm{mS}$ to $1 \mathrm{mS}$ ) is depicted in Fig. 8. We expect the theoretical FO tunability from $221 \mathrm{kHz}$ to $2.21 \mathrm{MHz}$ in all three cases $\left(B_{2}=2, B_{1} \leq 1, C_{1,2}=72 \mathrm{pF}, B_{2}=3, B_{1} \leq 2, C_{1,2}=102 \mathrm{pF}\right.$ and $\left.B_{2}=4, B_{1} \leq 3, C_{1,2}=125 \mathrm{pF}\right)$. The simulated frequency ranges achieve values from $211 \mathrm{kHz}$ to $2.02 \mathrm{MHz}$ $\left(B_{2}=2, B_{1} \leq 1, C_{1,2}=72 \mathrm{pF}\right), 213 \mathrm{kHz}$ to $2.07 \mathrm{MHz}\left(B_{2}=3\right.$, $\left.B_{1} \leq 2, C_{1,2}=102 \mathrm{pF}\right)$ and $211 \mathrm{kHz}$ to $2.09 \mathrm{MHz}\left(B_{2}=4\right.$, $\left.B_{1} \leq 3, C_{1,2}=125 \mathrm{pF}\right)$. Parasitic features of high-impedance nodes (additional capacitances of units of $\mathrm{pF}$ ) have significant effect on all cases for high frequency corner of observed FO range. However, we can see that second case (having larger values $C_{1,2}=102 \mathrm{pF}$ ) follows theoretical expectations more precisely than the first case $\left(C_{1,2}=72 \mathrm{pF}\right)$ and the third case $\left(C_{1,2}=125 \mathrm{pF}\right)$ even more precisely than the both previous cases. Better results (discriminability in Fig. 8) can be obtained for higher value of $B_{2}$. Nevertheless, for the same range of parameters controlling FO tuning $\left(g_{\mathrm{m}}, R_{\mathrm{X}}\right)$ all the time. On the other hand, gain values $\left(B_{2}\right.$ and $\left.B_{1}\right)$ increase.

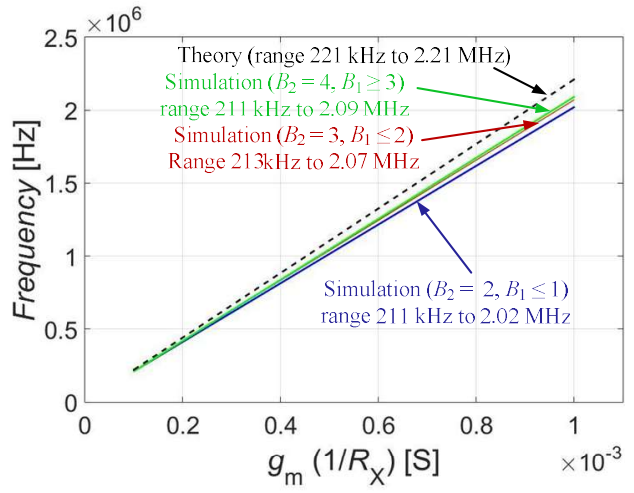

Fig. 8. Comparison of the dependency of the linear FO control for cases $B_{2}=2, B_{1} \leq 1, B_{2}=3, B_{1} \leq 2$ and $B_{2}=4, B_{1} \leq 3$ (for $g_{\mathrm{m}}=1 / R_{\mathrm{X}}$ ).

The feature of FO reinforcement introduced in this paper could be utilized in case of other oscillator designs (as long as the circuit topology is suitable for this approach). There are some points which need to be fulfilled so the feature of reinforcement can be applied more generally to other oscillators as well: a) given oscillator provides an independent control of FO and $\mathrm{CO}$ (many recent proposals offer this ability). b) based on the oscillator topology and the characteristic equation of the oscillator in our case, we suppose two electronically controllable parameters (current gains $B_{1}$ and $B_{2}$ in our case), both contained in the relation for $\mathrm{CO}$ and one of them contained in the relation for FO. The parameter in FO ( $B_{2}$ in our case) serves for shifting/boosting the FO range to higher frequencies while the other parameter $\left(B_{1}\right)$ ensures that $\mathrm{CO}$ is fulfilled in relation to the value of $B_{2}$. The ability of the independent control of FO and $\mathrm{CO}$ stays fulfilled. c) these electronically controllable parameters must be able to provide a great range of the obtainable values (higher values we can get, higher values of frequencies can be obtained, or higher values of working capacitances in the topology can be used while keeping the same operational range).
Oscillators with suitable topology can benefit from the FO reinforcement feature introduced in this paper by means of possibility to use higher values of working capacitances and thus decrease the effect of parasitic characteristics, higher obtainable frequencies while allowing the values of capacitances/transconductances/resistances to stay in reasonable range, or use of easily obtainable ranges of transconductances.

\section{CONCLUSION}

The $f_{0}$ of oscillations was tested for values of $g_{\mathrm{m}}\left(1 / R_{\mathrm{X}}\right)$ from 0.1 to $1 \mathrm{mS}$ providing testing range of $72 \mathrm{kHz} \rightarrow 723 \mathrm{kHz}$ (for $C_{1,2}=220 \mathrm{pF}$ ). The obtained range was $73 \mathrm{kHz} \rightarrow 684 \mathrm{kHz}$. The dependence of linear and nonlinear FO control can be compared in Figs. 4, 5 and 6. The FO reinforcement allows to obtain the same available theoretical range (for $g_{\mathrm{m}}\left(1 / R_{\mathrm{X}}\right)=0.1 \rightarrow 1 \mathrm{mS}$ ) $0.221 \mathrm{MHz} \rightarrow 2.21 \mathrm{MHz} \quad\left(B_{2}=2, \quad B_{1} \leq 1, \quad C_{1,2}=72 \mathrm{pF}\right)$ in comparison to $0.221 \mathrm{MHz} \rightarrow 2.21 \mathrm{MHz}\left(B_{2}=3, B_{1} \leq 2\right.$, $\left.C_{1,2}=102 \mathrm{pF}\right)$ and in comparison to $0.221 \mathrm{MHz}$ $\rightarrow 2.21 \mathrm{MHz}\left(B_{2}=4, B_{1} \leq 3, C_{1,2}=125 \mathrm{pF}\right)$. The obtained ranges from simulations yield $0.211 \mathrm{MHz} \rightarrow 2.02 \mathrm{MHz}$ without $\mathrm{FO}$ reinforcement (with $C_{1,2}=72 \mathrm{pF}$ ) and $0.221 \mathrm{MHz} \rightarrow 2.07 \mathrm{MHz} \quad$ (with $C_{1,2}=102 \mathrm{pF}$ ) and $0.221 \mathrm{MHz} \rightarrow 2.09 \mathrm{MHz}$ (with $C_{1,2}=125 \mathrm{pF}$ ) with the reinforcement. Thus, the feature of FO reinforcement allows usage of capacitors of higher values when parasitic features less affect the circuit. In other words, we obtained the same (slightly improved) range of FO tuning for larger values of $C_{1,2}$ without impact on parameters intended for $\mathrm{FO}$ control $\left(g_{\mathrm{m}}, R_{\mathrm{X}}\right)$. Similarly, the $B_{2}$ value can shift FO range to higher frequencies in case of nonlinear dependence of FO on $g_{\mathrm{m}}$ or $R_{\mathrm{X}}$ without change of $C_{1,2}$ as shown in Fig. 6 . These parameters have still the same range of control in all cases. Moreover, $B_{2}$ can be set to higher values as long as condition $B_{1} \leq B_{2}-1$ remains fulfilled. The verification of discussed hypothesis was the most important goal of this work.

\section{REFERENCES}

[1] R. Senani, D. R. Bhaskar, V. K. Singh, R. K. Sharma, Sinusoidal Oscillators and Waveform Generators using Modern Electronic Circuit Building Blocks, Switzerland: Springer International Publishing, 2016, pp. 1-622.

[2] A. Lahiri, "Current-mode variable frequency quadrature sinusoidal oscillator using two CCs and four passive components including grounded capacitors," Analog Integr. Circuits Signal Process, vol. 68, no. 1, 2011, pp. 129-131.

[3] A. M. Soliman "On the generation of CCII and ICCII oscillators from three Op Amps oscillator," Microelectron. J., vol. 41, no. 10, 2010, pp. 680-687.

[4] V. K. Singh, R. K. Sharma, A. K. Singh, D. R. Bhaskar, R. Senani, "Two New Canonic Single-CFOA Oscillators With Single Resistor Controls," IEEE Transactions on Circuits and Systems - II: Express Briefs, vol. 52, no. 12, 2005, pp. 860-864.

[5] A. Rodriguez-Vazquez, B. Linares-Barranco, J. Huertas, E. SanchezSinencio, "On the Design of Voltage-Controlled Sinusoidal Oscillators Using OTA's" IEEE Transactions on Circuits and Systems, vol. 37, no. 2, 1990, pp. 198-211.

[6] S-H. Tu, Y-S. Hwang, J-J. Chen, A. M. Soliman, C-M. Chang, "OTA-C arbitrary-phase-shift oscillators", IEEE Transactions on Instrumentation and Measurement, vol. 61, no. 8, 2012, pp. 23052319.

[7] W. Jaikla, M. Siripruchyanun, A. Lahiri, "Resistorless dual-mode quadrature sinusoidal oscillator using a single active building block," Microelectron. J., vol. 42, no. 1, 2011, pp. 135-140.

[8] W. Jaikla and P. Prommee, "Electronically tunable current-mode multiphase sinusoidal oscillator employing CCCDTA-based allpass 
filters with only grounded passive elements," Radioengineering, vol. 20, no. 3, 2011, pp. 594-599.

[9] Y. Li, "Systematic derivation for quadrature oscillators using CCCCTAs," Radioengineering, vol. 24, no. 2, 2015, pp. 535-543.

[10] D. Biolek, A. Lahiri, W. Jaikla, M. Siripruchyanun, and J. Bajer, "Realization of electronically tunable voltage-mode/current-mode quadrature sinusoidal oscillator using ZC-CG-CDBA," Microelectron. J., vol. 42, no. 10, 2011, pp. 1116-1123.

[11] R. Sotner, J. Jerabek, N. Herencsar, J.-W. Horng, K. Vrba, and T. Dostal, "Simple oscillator with enlarged tunability range based on ECCII and VGA utilizing commercially available analog multiplier," Measurement Science Review, vol. 16, no. 2, 2016, pp. 35-41.

[12] D. Biolek, R. Senani, V. Biolkova, Z. Kolka, "Active Elements for Analog Signal Processing: Classification, Review, and New Proposals," Radioengineering, vol. 17, no. 4, 2008, pp. 15-32.

[13] E. Sanchez-Sinencio, J. Silva-Martinez, "CMOS transconductance amplifiers, architectures and active filters: a tutorial," IEE Proc. Circ. Dev. Systems, vol. 147, no. 1, 2000, pp. 3-12.

[14] R. Sotner, J. Jerabek, N. Herencsar, J. Petrzela, "Methods for Extended Tunability in Quadrature Oscillators Based on Enhanced Electronic Control of Time Constants," IEEE Transactions on Instrument. and Measurement, vol. 67, no. 6, 2018, pp. 1495-1505.

[15] R. Sotner, J. Jerabek, J. Petrzela, N. Herencsar, R. Prokop, K. Vrba, "Second-Order Simple Multiphase Oscillator Using Z-Copy Controlled-Gain Voltage Differencing Current Conveyor" Elektronika Ir Elektrotechnika, vol. 20, no. 9, 2014, pp. 13-18.
[16] R. Sotner, J. Jerabek, N. Herencsar, J. Horng, K. Vrba, "Electronically Linearly Voltage Controlled Second-Order Harmonic Oscillator With Multiples of pi/ 4 Phase Shifts," In Proc. of the 38th International Conference on Telecommunication and Signal Processing, Berlin, Germany, 2014. pp. 708-712.

[17] R. Sotner, J. Jerabek, N. Herencsar, K. Vrba, T Dostal, "Features of multi-loop structures with OTAs and adjustable current amplifier for second-order multiphase/quadrature oscillators," AEU - International Journal of Electronics and Communications, vol, 69, 2015, pp. 814 822.

[18] R. Sotner, J. Jerabek, N. Herencsar, K. Vrba, "Design of the simple oscillator with linear tuning and $\mathrm{pi} / 4$ phase shift based on emulator of the modified current differencing unit." IEICE Electronics Express, vol. 12 , no. 19,2015 , pp. 1-7.

[19] Intersil (Elantec), EL2082 CN Current-mode multiplier (datasheet), 1996 , accessible on www: http://www.intersil.com/data/fn/fn7152.pdf.

[20] Linear Technology, LT1228 Current Feedback Amplifier with DC Gain Control (datasheet), accessible on http://cds.linear.com/docs/en/datasheet/1228fd.pdf.

[21] Analog Devices, AD844 Operational Amplifier (datasheet), 1989, accessible on http://www.analog.com/media/en/technicaldocumentation/data-sheets/AD844.pdf.

[22] Intersil (Elantec), EL4083 CN Current-mode multiplier (datasheet), 1996, accessible www.intersil.com/content/dam/Intersil/documents/el40/el4083.pdf 